\title{
Direct ophthalmoscopy should be taught to undergraduate medical students-No
}

Eye (2015) 29, 990-991; doi:10.1038/eye.2015.91; published online 5 June 2015

Experienced physicians using the direct ophthalmoscope lack confidence and often fail to notice important abnormalities. ${ }^{1-3}$ Missed or delayed detection of ophthalmic signs can result in harm to patients: undetected disc swelling could lead to loss of vision from persistently raised intracranial pressure; an undiagnosed retinal artery occlusion could prevent potentially life-saving risk factor modification in a patient at risk of stroke; failure to spot Roth spots could delay the diagnosis of bacterial endocarditis. Ophthalmology-but not direct ophthalmoscopy-is an essential part of undergraduate medical education. The diagnosis of many systemic conditions can be made or assisted by the identification of ophthalmic features, and knowledge of ophthalmology in relation to acute medical and surgical problems in invaluable for any doctor. However, these signs must first be detected before they can be acted upon. The time spent in ophthalmology by undergraduate medical students in the UK is limited, with most students spending 5-10 days of their entire training in compulsory ophthalmology education, ${ }^{4}$ and trends across the developed world predict further reduction, ${ }^{5}$ so it is vitally important that these precious hours are used wisely to ensure tomorrow's doctors are best equipped to serve their patients. Considering such time restraints, rather than struggling to wield a direct ophthalmoscope in busy clinics, further clinical teaching is more of a priority for the modern medical student, which would enhance their ability to interpret signs from fundus images that can be captured by other means.

Expecting medical students to become competent in direct ophthalmoscopy in the aforementioned time frame is unrealistic. Although they may be able to gain confidence in the basic setup and handling of the equipment, reaching a standard where their findings can be relied upon is wildly optimistic, particularly when you consider that direct ophthalmoscopy is often considered a problem station by senior Ophthalmic Specialist Trainees in the clinical diet of the Part 2 FRCOphth examination. ${ }^{6}$ Despite specific changes to the foundation curriculum aimed at improving the quality and completeness of neurological examination in two Birmingham trusts, only one in five doctors felt confident in recognising papilloedema (ie, disc swelling) by the end of their foundation year and $71 \%$ had used an ophthalmoscope fewer than ten times in the preceding 6 months. ${ }^{1}$ Furthermore, a 1997 survey found that $43 \%$ of UK general practitioners lacked confidence in using the direct ophthalmoscope. ${ }^{2}$

Fundus examination is performed too infrequently by general physicians and emergency doctors, and, when it is carried out, the quality is poor. In the first phase of the FOTO-ED study, ${ }^{3}$ only $14 \%$ of 350 patients who presented to an emergency department with symptoms or signs warranting fundus examination (acute visual change, focal neurological deficit, headache or diastolic blood pressure $>120 \mathrm{~mm} \mathrm{Hg}$ ) had a fundoscopic examination by an emergency physician. Nonmydriatic fundus photography identified new ocular pathology in 33 patients, but in only five of these 33 patients was ophthalmoscopy performed by the attending doctor-in all five cases the examination was recorded as 'normal', that is, the relevant ocular abnormalities were missed.

Technical barriers may be the main issue behind the under-utilisation of direct ophthalmoscopy. The TOTeMS study ${ }^{7}$ concluded that students preferred fundus photographs for both learning and examining the ocular fundus, with $70 \%$ saying they would prefer to have fundus photographs instead of using the ophthalmoscope during upcoming clinical rotations. When tested, students' answers were significantly more accurate when interpreting fundus photographs than when performing ophthalmoscopy. In the second phase of the FOTOED study ${ }^{8}$, emergency department physicians had access to non-mydriatic fundus photographs, which 
they used far more readily than the direct ophthalmoscope (photos were reviewed in 68\% of patients with headache, focal neurological deficit, visual change, or diastolic blood pressure $>120 \mathrm{~mm} \mathrm{Hg}$; whereas fundoscopy was performed in only $14 \%$ of patients presenting with similar symptoms/ signs in the original study ${ }^{3}$ ).

Unlike direct ophthalmoscopy, fundus photography is easy to perform. Allied health professionals, previously naïve to the technology, are able to capture good quality images with $<30$ min training. ${ }^{9}$ Ophthalmoscopes are expensive and often poorly maintained in hospital departments. ${ }^{1}$ Fundus cameras are becoming cheaper and more portable: the Peek Vision project (www.peekvision.org) uses a 3D-printed adapter to allow a smartphone to be used as a fundus camera for a total cost of under $\$ 500$, which produces images of comparable quality to that of standalone cameras costing fifty times more.

The routine use of fundus photography also has advantages related to telemedicine $\mathrm{e}^{10-12}$ and record keeping, and it is the method of choice for diabetic eye screening. All too often, referrals from emergency departments to ophthalmology are concluded with a statement such as 'I couldn't see any obvious abnormalities with the ophthalmoscope, but it was difficult...' Digital images can be emailed in a secure fashion between departments, either to support a referral or in order to obtain specialist advice from an ophthalmologist.

Fundus examination needs to be part of the complete physical examination as per the GMC and the Foundation Programme Curriculum. ${ }^{13}$ Simply attempting fundoscopy is not good enough - the examination needs to be reliable and accurate; the documentation of an erroneous 'normal' fundus examination could certainly lead to harm. There are two options to improve the quality of the examination: firstly commit more time and even more resources to teaching, and regularly assessing, direct ophthalmoscopy, which has been shown to be performed poorly even by experienced clinicians; or, secondly, to routinely perform fundus photography which can be reviewed by the attending medical team and facilitate onward referral if required. The second approach has clear advantages supported by evidence from the modern medical literature: it will empower non-ophthalmologists by allowing them to overcome that most significant hurdle of obtaining a useful fundal view; and, ultimately and most importantly, improve patient safety and quality of care.

\section{Conflict of interest}

The authors declare no conflict of interest. NVC is a consultant for Allergan, Bayer, Novartis, Quantel Medical and Pfenex but none of these consultancy agreements have any conflict with the subject matter in this article.

\section{References}

1 Nicholl DJ, Yap CP, Cahill V, Appleton J, Willetts E, Sturman S. The TOS study: can we use our patients to help improve clinical assessment? J R Coll Physicians Edinb 2012; 42(4): 306-310.

2 Shuttleworth N, Marsh W. How effective is undergraduate and postgraduate teaching in ophthalmology? Eye 1997; 11 (5): 744-750.

3 Bruce BB, Lamirel C, Wright DW, Ward A, Heilpern KL, Biousse $\mathrm{V}$ et al. Nonmydriatic ocular fundus photography in the emergency department. N Engl J Med 2011; 364: . 387-389.

4 Baylis O, Murray PI, Dayan M. Undergraduate ophthalmology education - a survey of UK medical schools. Med Teach 2011; 33(6): 468-471.

5 Mottow-Lippa L. Ophthalmology in the medical school curriculum: reestablishing our value and effecting change. Ophthalmology 2009; 116(7): 1235-1236 e1.

6 The Royal College of Ophthalmologists. Examination Reports (Internet). (cited 21 October 2014, Retrieved from http://www.rcophth.ac.uk/page.asp?section= 118\&sectionTitle=Examination+Reports.

7 Kelly LP, Garza PS, Bruce BB, Graubart EB, Newman NJ, Biousse V. Teaching ophthalmoscopy to medical students (the TOTeMS study). Am J Ophthalmol 2013; 156(5): 1056-1061 e10.

8 Bruce BB, Thulasi P, Fraser CL, Keadey MT, Ward A, Heilpern KL et al. Diagnostic accuracy and use of nonmydriatic ocular fundus photography by emergency physicians: phase II of the FOTO-ED study. Ann Emerg Med 2013; 62(1): 28-33 e1.

9 Lamirel C, Bruce BB, Wright DW, Delaney KP, Newman NJ, Biousse V. Quality of nonmydriatic digital fundus photography obtained by nurse practitioners in the emergency department: the FOTO-ED study. Ophthalmology 2012; 119(3): 617-624.

10 Ausayakhun S, Skalet AH, Jirawison C, Ausayakhun S, Keenan JD, Khouri $C$ et al. Accuracy and reliability of telemedicine for diagnosis of cytomegalovirus retinitis. Am J Ophthalmol 2011; 1526: 1053-1058 e1.

11 Chiang MF, Wang L, Kim D, Scott K, Richter G, Kane S et al. Diagnostic performance of a telemedicine system for ophthalmology: advantages in accuracy and speed compared to standard care. AMIA Annu Symp Proc, American Medical Informatics Association 2010; 2010: 111-115.

12 Scott KE, Kim DY, Wang L, Kane SA, Coki O, Starren J et al. Telemedical diagnosis of retinopathy of prematurity. Ophthalmology 2008; 115(7): 1222-1228 e3.

13 The Foundation Programme Curriculum (Internet). (cited 21 October 2014). Retrieved from http://www. foundationprogramme.nhs.uk/pages/home/curriculumand-assessment/curriculum2012.

RMJ Purbrick ${ }^{1}$ and NV Chong ${ }^{2}$

${ }^{1}$ Sussex Eye Hospital, Brighton, UK

${ }^{2}$ Oxford Eye Hospital, Oxford University Hospitals, Oxford, UK

Correspondence: NV Chong, Ophthalmology, Oxford Eye Hospital, Oxford University Hospitals, Headley Way, Headington, Oxford OX3 9DU, UK Tel: +44 (0)1865 234 736; Fax: +44 (0)1865 234515 . E-mail: victor@eretina.org 